\title{
ON DYNAMICS OF MOSTLY CONTRACTING DIFFEOMORPHISMS.
}

\author{
DMITRY DOLGOPYAT
}

\begin{abstract}
Mostly contracting diffeomorphisms are the simplest examples of robustly nonuniformly hyperbolic systems. This paper studies the mixing properties of mostly contracting diffeomorphisms.
\end{abstract}

\section{INTRODUCTION.}

This paper treats a class of partially hyperbolic systems with nonzero Lyapunov exponents.

Before stating our result let us recall some recent work motivating our research. In recent years there were several advances in understanding of statistical properties of weakly hyperbolic dynamical systems.

On one hand L.-S. Young developed quantitative Pesin theory in $[38,39]$. Among other things she proved that if a diffeomorphism $f$ has a Pesin set $\Lambda$ such that the distribution of the return time to $\Lambda$ has an exponentially decaying tail and if $f$ has no discrete spectrum then it is exponentially mixing. This theory was applied to a number of examples in the above mentioned papers as well as in $[3,10]$.

On the other hand M. Grayson, C. Pugh and M. Shub showed ([13, $27,28]$ ) that partial hyperbolicity can give raise to a good ergodic behavior in a robust way. Further examples of systems satisfying their criteria can be found in $[7,8,16,20,37]$.

These results lead to the natural question if there is an open set of (partially hyperbolic) systems satisfying the conditions of Young's theory with uniform bounds. This question was addressed in a number of papers $[1,2,4,32,36]$. Our paper also fits into this framework.

Let us give a few definitions. Let $f$ be a diffeomorphism of a smooth manifold $X$ and let $\nu$ be an ergodic $f$-invariant measure. We call $\nu$ an $S R B$-measure for $f$ if there is a subset $Y(\nu) \subset X$ of positive Lebesgue measure such that for almost all $y \in Y$ for any continuous function $A$ $\frac{1}{n} \sum_{j=0}^{n-1} A\left(f^{j} x\right) \rightarrow \nu(A) . Y(\nu)$ is called basin of attraction of $\nu$. Certainly the question of existence of SRB-measures and their dependence on 
parameters is quite important in smooth ergodic theory. We say that $f$ has a global attractor if there is only one SRB-measure whose basin is all of $X$. (Our use of the word attractor follows that of [35, 27]. More precisely, $\nu$ or $\operatorname{supp}(\nu)$ should be called a stochastic attractor because it describes the statistical properties of large iterations of $f$. For a more topological approach see [19].) Let $\mathcal{S}$ be a subset of $\operatorname{Diff}_{r}(X), r>1$ endowed with some topology (think of $\mathcal{S}$ as a parameter space) and let $f \in \mathcal{S}$. We call $f$ statistically stable in $\mathcal{S}$ if any diffeomorphism $g$ in some neighborhood of $f$ in $\mathcal{S}$ has a finite number of SRB-measures $\nu_{1}(g), \nu_{2}(g) \ldots \nu_{k}(g)$, the maps $g \rightarrow \nu_{j}(g)$ are continuous and the union of basins of $\nu_{j}(g)$ is all of $X$. If $k=1$ we call $f$ strongly statistically stable. Below we deal with the case when $\mathcal{S}=\operatorname{Diff}_{2}(X)$ with uniform $C^{2}$-topology.

In this note we provide some sufficient conditions for statistical stability as well as for other good statistical properties. Our main results are the following.

Theorem I. Let $f$ be partially hyperbolic dynamically coherent u-convergent mostly contracting diffeomorphism of a three-dimensional manifold $X$. Then

(a) $f$ has a global attractor $\nu$;

(b) for any $\gamma>0$ there are constants $C, \zeta<1$ such that if $A, B \in$ $C^{\gamma}(X)$ then for positive $n$

$$
\begin{gathered}
\left|\int B(x) A\left(f^{n} x\right) d x-\nu(A) \int B(x) d x\right| \leq C \zeta^{n}\|A\|_{\gamma}\|B\|_{\gamma}, \\
\left|\int B(x) A\left(f^{n} x\right) d \nu(x)-\nu(A) \nu(B)\right| \leq C \zeta^{n}\|A\|\left\|_{\gamma}\right\| B \|_{\gamma}
\end{gathered}
$$

(c) f has non-zero Lyapunov exponents.

Remark. Parts (a) and (c) of this theorem were established before in [4] for a larger class of systems. It follows from (c) and the results of $[11,21]$ that the system $(X, f, \nu)$ is a Bernoulli shift.

Remark. In fact, we prove more than (a). Namely we show that the image under $f^{n}$ of any unstable leaf becomes equidistributed. In [12] we proved that diffeomorphisms having this property satisfy many classical limit theorems of probability theory.

Theorem II. Let $f$ be as in Theorem I. If in addition $f$ is stably dynamically coherent then $f$ is strongly statistically stable. More precisely, there exists a neighborhood $\mathcal{O}(f) \subset \operatorname{Diff}_{2}(X)$ such that any $g \in \mathcal{O}$ satisfies the conditions of Theorem $I$ and the constants $C, \zeta$ in Theorem $I(b)$ can be chosen uniformly in $\mathcal{O}(f)$. In particular if 
$\nu_{g}$ is the $S R B$ measure for $g$ then for any $\gamma>0$ the map $g \rightarrow \nu_{g}$ $\mathcal{O}(f) \rightarrow\left(C^{\gamma}(X)\right)^{*}$ is Holder continuous.

See Sections 2, 3 for the definition of the terms appearing in the formulation of this theorem. In Section 4 we show that for mostly contracting diffeomorphisms the second forward Lyapunov exponent of almost every point is negative and prove large deviation estimates for the exceptional set. This is done by certain submartingale estimates more common in the theory of stochastic differential equations. In Section 5 we recall the construction of u-Gibbs measures [26] and show that in our situation they are SRB measures. The uniqueness of SRB measures is treated in Section 6-9. In Section 6 we recall the coupling method of L.-S. Young. In Section 7 we describe coupling algorithm for our system. The properties of this algorithm are studied in Sections 8 and 9. The proofs of the main theorems are completed in Section 10. In Sections 11 and 12 we discuss some examples. Final remarks and some open questions are presented in Section 13.

Remark. Independently and slightly earlier A. Castro [9] proved a result similar to our Theorem I. However, because of some technical assumptions in his paper it is not clear if his result can be applied to the examples of Section 12.

Acknowledgment. I thank V. Nitica, C. Pugh and M. Ratner for useful discussions. I first learned about mostly contracting systems during Ergodic Theory and Statistical Mechanics Seminar at Princeton University where a random version of this property was discussed. I thank all the participants of that seminar and especially K. Khanin, A. Mazel and Ya. Sinai for introducing me to this subject. In a previous version of my paper I imposed a strong regularity requirement on the unstable foliation to prove part (A) of Lemma 6.1. I am grateful to C. Bonatti and M. Viana for explaining me that Pesin theory can be used to verify this property (see Lemma 8.1). This work is supported by Miller Institute for Basic Research in Science.

\section{Partial hyperbolicity.}

In this and the next sections we describe the properties of $f$ which appear in the statement of Theorem I. As it was mentioned in the introduction $f$ is a diffeomorphism of $X^{3}$. We also assume that $f$ is partially hyperbolic and stably dynamically coherent. Thus the tangent bundle of $X$ is the sum of three continuous one dimensional subbundles $E_{u}, E_{c}$ and $E_{s}$ such that

$$
e^{\lambda_{1}} \leq\left(d f \mid E_{s}\right) \leq e^{\lambda_{2}}
$$




$$
\begin{gathered}
e^{\lambda_{3}} \leq\left(d f \mid E_{c}\right) \leq e^{\lambda_{4}} \\
e^{\lambda_{5}} \leq\left(d f \mid E_{u}\right) \leq e^{\lambda_{6}}
\end{gathered}
$$

where $\lambda_{1} \leq \lambda_{2}<\lambda_{3} \leq \lambda_{4}<\lambda_{5} \leq \lambda_{6}$ and $\lambda_{2}<0, \lambda_{5}>0$.

$E_{u}$ and $E_{s}$ are always integrable so they are tangent to $f$ invariant foliations: unstable $\left(W^{u}\right)$ and stable $\left(W^{s}\right)$. Dynamical coherence means that $E_{c}, E_{c} \oplus E_{u}$ and $E_{c} \oplus E_{s}$ are also are tangent to $f$-invariant foliations which are called central $\left(W^{c}\right)$, center-unstable $\left(W^{c u}\right)$ and center-stable $\left(W^{c s}\right)$ respectively, and that $W^{c}$ subfoliates both $W^{c u}$ and $W^{c s}$. (In fact only unique integrability of $E_{c} \oplus E_{s}$ is used in the paper.) Stable dynamical coherence means that any $g$ close to $f$ is also dynamically coherent. The openness of these conditions was studied in [14]. Namely partial hyperbolicity is open. It is unknown if dynamical coherence is open but if the center foliation of $f$ is $C^{1}$ then $f$ is stably dynamically coherent.

Let $\mathcal{V}$ be the set of all unstable curves of lengths between 1 and $2 . \mathcal{V}$ is a Markov family in the sense that $\forall V \in \mathcal{V}$ there is a finite set $\left\{\tilde{V}_{j}\right\}$ of elements of $\mathcal{V}$ such that $f V=\bigcup_{j} \tilde{V}_{j}$. More generally for any unstable curve $U$ of length greater than one there is a finite set $\left\{V_{j}\right\}$ such that $V_{j} \in \mathcal{V}$ and

$$
U=\bigcup_{j} V_{j}
$$

We call (4) Markov decomposition of $U$. We will use (4) for $U=f^{n} V$ where $V \in \mathcal{V}, n>0$.

We call $f$ u-convergent if $\forall \varepsilon \exists n>0 \forall V_{1}, V_{2} \in \mathcal{V} \exists x_{j} \in V_{j}$ such that $d\left(f^{n} x_{1}, f^{n} x_{2}\right) \leq \varepsilon$. Later on we show that u-convergence is open among mostly contracting diffeomorphisms.

\section{Mostly CONTRACTING Systems.}

The assumptions of Section 2 are routine partial hyperbolicity assumptions. The next property guarantees that $f$ is non-uniformly hyperbolic. In order to formulate it we need to recall the definition of canonical densities on $W^{u}$. We would like to study SRB-measure for $f$. A priori we do not know if it exists, but if it does then its conditional densities on $W^{u}$ are given by [26]. Fix a Riemann structure on $X$. It induces a metric on $W^{u}$-fibers. Let $V$ be an interval inside $W^{u}$. For $z_{1}, z_{2} \in V$ let

$$
\rho\left(z_{1}, z_{2}\right)=\prod_{j=0}^{\infty} \frac{\left(d f^{-1} \mid E_{u}\right)\left(f^{-j} z_{1}\right)}{\left(d f^{-1} \mid E_{u}\right)\left(f^{-j} z_{2}\right)}
$$


Fix some $z_{0} \in V$ and let $\rho_{V}(z)=C \rho\left(z, z_{0}\right)$ where $C=\left(\int_{V} \rho\left(z, z_{0}\right) d z\right)^{-1}$. Since $\rho\left(z, z_{0}^{\prime}\right)=\rho\left(z, z_{0}\right) \rho\left(z_{0}, z_{0}^{\prime}\right)$ this definition actually does not depend on $z_{0}$. Also if $W=f V, y=f z$ then

$$
\begin{gathered}
\rho_{V}(z) d z=C_{V} \rho\left(z, z_{0}\right) d z=C_{V} \rho\left(z, z_{0}\right)\left(d f^{-1} \mid E_{u}\right)(y) d y= \\
\tilde{C}_{V} \rho\left(z, z_{0}\right) \frac{\left(d f^{-1} \mid E_{u}\right)(y)}{\left(d f^{-1} \mid E_{u}\right)\left(y_{0}\right)} d y=C_{W} \rho\left(y, y_{0}\right) d y .
\end{gathered}
$$

Thus if $A$ is continuous then

$$
\int_{V} A(f z) \rho_{V}(z) d z=\int_{f V} A(y) \rho_{f V}(y) d y
$$

Our last assumption is the following. There is a positive constant $\alpha_{0}$ such that for any $V \in \mathcal{V}$

$$
\int_{V} \rho_{V}(x)\left(\ln \left(d f \mid E_{c}\right)(x)\right) d x \leq-\alpha_{0}<0
$$

We call $f$ mostly contracting if some positive power of it satisfies (6). In the proof of the Theorem I we assume, as we may, that $f$ itself satisfies (6).

Remark. Condition (6) is $C^{2}$-open. Indeed by standard theory ([14]) the map CENTER $(f)=\left(d f \mid E_{c}\right)(x)$ is continuous: $\operatorname{Diff}_{2}(X) \rightarrow C(X)$. Let $V(x, f, t)$ denote $f$-unstable curve of length $t$ centered at $x$. Let $V(x, f, t)(\tau)$ be the arclength parameterization of $\tau$. Denote $\mathbf{T}=\{(t, \tau)$ : $1 \leq t \leq 2,0 \leq \tau \leq t\}$. Then the map Density $(f)=\rho_{V(x, f, t)}(V(x, f, t)(\tau))$ is continuous: $\operatorname{Diff}_{2}(X) \rightarrow C(X \times \mathbf{T})$ since $\rho$ is a uniform limit of continuous functions. CENTER is also continuous in $C^{1}$-topology, but DENSITY is not because convergence in (5) may fail to be uniform in $f$ (cf. [23]). Thus it is unclear if mostly contractiveness is $C^{1}-$ open.

\section{LARGE DEVIATIONS.}

Here we prove

Theorem 4.1. $\exists C_{1}, s>0, \theta_{1}<1$ such that $\forall V \in \mathcal{V} \forall n>0$

$$
\int_{V} \rho_{V}(x)\left(d f^{n} \mid E_{c}\right)^{s}(x) d x \leq C_{1} \theta_{1}^{n}
$$

The proof consists of a number of lemmas.

\section{Lemma 4.2.}

$$
\forall n>0 \quad \int_{V} \rho_{V}(x) \ln \left(d f^{n} \mid E_{c}\right)(x) d x \leq-n \alpha_{0}
$$


Proof. We have

$$
\begin{gathered}
\int_{V} \rho_{V}(x) \ln \left(d f^{n} \mid E_{c}\right)(x) d x= \\
\int_{V} \rho_{V}(x) \ln \left(d f \mid E_{c}\right)(x) d x+\int_{f V} \rho_{f V}(y) \ln \left(d f^{n-1} \mid E_{c}\right)(y) d y .
\end{gathered}
$$

Let $f V=\bigcup_{j} V_{j}$ be an almost Markov decomposition. Then the second term equals

$$
\sum_{j} c_{j} \int_{V_{j}} \rho_{V_{j}}(y) \ln \left(d f^{n-1} \mid E_{c}\right)(y) d y
$$

where $c_{j}=\int_{f^{-1} V_{j}} \rho_{V}(x) d x$. By induction

$$
\int_{V_{j}} \rho_{V_{j}}(y) \ln \left(d f^{n-1} \mid E_{c}\right)(y) d y \leq-(n-1) \alpha_{0} .
$$

Summation over $j$ proves the lemma.

The following distortion bound is standard (see, for example [4], Lemma $3.3)$.

Proposition 4.3. There is a constant $C$ so that $\forall n>0 \forall V \in \mathcal{V}$ $\forall x_{1}, x_{2} \in f^{-n} V$

$$
\left|\ln \left(d f^{n} \mid E_{c}\right)\left(x_{1}\right)-\ln \left(d f^{n} \mid E_{c}\right)\left(x_{2}\right)\right| \leq C .
$$

If $A$ is a continuous function and $U$ is a piece of unstable manifold we write $\|A\|_{U}=\max _{x \in U}|A(x)|$.

Corollary 4.4. There exists $\alpha_{1}$ such that if $n$ is large enough then for any $V \in \mathcal{V}$ for any Markov decomposition $f^{n} V=\bigcup_{j} V_{j}$ the following holds. Let $U_{j}=f^{-n} V_{j}, c_{j}=\int_{U_{j}} \rho_{V}(x) d x$. Then

$$
\sum_{j} c_{j} \ln \left\|\left(d f^{n} \mid E_{c}\right)\right\|_{U_{j}} \leq-\alpha_{1}
$$

Changing if necessary $f \rightarrow f^{n}$ we can assume that this is true for $n=1$. Under this assumption we have

Lemma 4.5. If $s$ is small enough there is a constant $\theta_{2}(\gamma)<1$ such that under the conditions of the previous corollary

$$
\sum_{j} c_{j}\left\|d f \mid E_{c}\right\|_{U_{j}}^{s} \leq \theta_{2}
$$

Proof. Regard the LHS as a function $r(s)$. Then $r(0)=1, \frac{d r}{d s}(0) \leq-\alpha_{1}$, $\left|\frac{d^{2} r}{d s^{2}}(0)\right| \leq$ Const.

Repeating the argument of Lemma 4.2 we obtain 
Corollary 4.6. For any $n>0$ there is a Markov decomposition $f^{n} V=$ $\bigcup_{j} V_{j}$ such that if $U_{j}=f^{-n} V_{j}, c_{j}=\int_{U_{j}} \rho_{V}(x) d x$ then

$$
\sum_{j} c_{j}\left|d f^{n}\right| E_{c} \|_{U_{j}}^{s} \leq \theta_{2}^{n}
$$

Proof. By induction. Suppose that (8) is valid for all $n \leq n_{0}-1$. Let $f V=\bigcup_{j} V_{j}$ be a Markov decomposition. By inductive assumption $\forall j$ there is a Markov decomposition $f^{n_{0}-1} V_{j}=\bigcup_{k} V_{j k}$ satisfying (8). Let $U_{j}=f^{-1} U_{j}, U_{j k}=f^{-n_{0}} V_{j k}, b_{j}=\int_{U_{j}} \rho_{V}(x) d x, c_{j k}=\int_{U_{j k}} \rho_{U_{j}}(x) d x$. Then $f^{n_{0}} V=\bigcup_{j k} V_{j k}$ is Markov and

$$
\begin{gathered}
\sum_{j k} \int_{f^{-n_{0} V_{j k}}} \rho_{V}(x)\left\|\left(d f^{n_{0}} \mid E_{c}\right)\right\|_{U_{j k}}^{s} d x= \\
\sum_{j k} b_{j} c_{j k} \|\left.\left(d f^{n_{0}} \mid E_{c}\right)\right|_{U_{j k}} ^{s} \leq \\
\sum_{j} b_{j}\left\|\left(d f \mid E_{c}\right)\right\|_{U_{j}}^{s} \sum_{k} c_{j k}\left(\left\|d f^{n_{0}-1} \mid E_{c}\right\|_{f U_{j k}}\right)^{s} \leq \\
\sum_{j} b_{j} \|\left.\left(d f \mid E_{c}\right)\right|_{U_{j}} ^{s} \theta_{2}^{n_{0}-1} \leq \\
\theta_{2}^{n_{0}} .
\end{gathered}
$$

This Corollary proves Theorem 4.1 since for any Markov decomposition $f^{n} V=\bigcup_{j} V_{j}$ LHS of (8) majorates LHS of (7).

\section{TRANSFER OPERATOR.}

Now we recall the general method of the construction of SRB-measures for partially hyperbolic systems ([26]). SRB measures are obtained as forward iteration limits of suitable measures. Here we describe the set of the initial measures. Fix some $R$. Let $E_{1}(R)$ be the set of measures of the form

$$
l(A)=\int_{V} A(z) e^{G(z)} \rho_{V}(z) d z
$$

where $V \in \mathcal{V}, l(1)=1$ and $\left|G\left(z_{1}\right)-G\left(z_{2}\right)\right| \leq R d^{\gamma}\left(z_{1}, z_{2}\right)$. (Those three conditions also guarantee that $G$ is uniformly bounded.) Let $E_{2}(R)$ be the convex hall of $E_{1}(R)$ and $E(R)$ be the closure of $E_{2}(R)$. The family $\{E(R)\}$ is continuous from above in the sense that $E\left(R_{0}\right)=$ $\bigcap_{R>R_{0}} E(R)$. (This follows from the fact that $E_{1}\left(R_{0}\right)=\bigcap_{R>R_{0}} E_{1}(R)$.)

Let $\mathcal{T}(l)(A)=l(A \circ f)$.

Proposition 5.1. $\mathcal{T}: E(R) \rightarrow E\left(R e^{-\lambda_{5} \gamma}\right)$. 
Proof. If $l \in E_{1}(R)$. Then

$$
(\mathcal{T} l)(A)=\int_{V} e^{G(z)} A(f z) \rho_{V}(z) d z=\int_{f V} e^{\left(G \circ f^{-1}\right)(y)} A(y) \rho_{f V}(y) d y .
$$

Let $f V=\bigcup_{j} V_{j}$, be a Markov decomposition then $\mathcal{T} l=\sum c_{j} l_{j}$, where

$$
l_{j}(A)=\int_{V_{j}} e^{\left(G \circ f^{-1}\right)(y)} A(y) \rho_{V_{j}}(y) d y .
$$

Also $\left|\left(G \circ f^{-1}\right)\left(y_{1}\right)-\left(G \circ f^{-1}\right)\left(y_{2}\right)\right| \leq R e^{-\lambda_{5} \gamma} d^{\gamma}\left(y_{1}, y_{2}\right)$. Thus $\mathcal{T}$ : $E_{1}(R) \rightarrow E_{2}\left(R e^{-\lambda_{5} \gamma}\right)$.

Since $E(R)$ is a convex compact set Proposition 5.1 implies that there is an $f$-invariant measure in $E(R)$ which moreover belongs to $\bigcap_{R>0} E(R)=$ $E(0)$ (this is also proven in [26]).

Proposition 5.2. Any $f$-invariant measure $\nu \in E(0)$ has two negative Lyapunov exponents.

Proof. By (6) for any $l \in E(0) l\left(\ln \left(d f \mid E_{c}\right)\right)<-\alpha_{0}$.

Proposition 5.2 and Lemma 13 of [26] guarantee that $\nu$ satisfies the conditions of Theorem 3 of [27] and so it is a SRB measure. (Another proof of this fact is given in Section 10.)

\section{Coupling argument.}

We now pass to the uniqueness of $\nu$. It is established via the coupling argument of [39]. We want to show that for large $n$ for any $l_{1}, l_{2} \in$ $E(R) \mathcal{T}^{n}\left(l_{1}\right)$ is close to $\mathcal{T}^{n}\left(l_{2}\right)$. First we consider the case when $l_{1}$ and $l_{2}$ are in $E_{1}(0)$, say $l_{j}(A)=\int_{V_{j}} A(x) \rho_{V_{j}}(x) d x$. The idea is to divide $f^{n} V_{j}$ into small pieces and pair the pieces of $f^{n} V_{1}$ to $f^{n} V_{2}$ so that the members of the pair are very close to each other. However since $f^{n}$ gives different weights to different pieces of $f^{n} V_{j}$ it is more convenient to regard unstable curves as 1-chains so that the heavier ones can be split into several pieces each one being paired to a different partner.

Let us now give a formal statement. Denote $Y_{j}=V_{j} \times[0,1]$. Equip $Y_{j}$ with the measure $d m_{j}(x, t)=\rho_{V_{j}}(x) d x d t$. The heart of the coupling method is the following result the proof of which occupies the next three sections.

Lemma 6.1. There is a measure preserving map $\tau: Y_{1} \rightarrow Y_{2}$, a function $R: Y_{1} \rightarrow \mathbb{N}$ and constants $C_{1}, C_{2}>0, \rho_{1}<1, \rho_{2}<1$ such that

(A) If $\left(x_{2}, t_{2}\right)=\tau\left(x_{1}, t_{1}\right)$ then for $n \geq R\left(x_{1}, t_{1}\right)$

$$
d\left(f^{n} x_{1}, f^{n} x_{2}\right) \leq C_{1} \rho_{1}^{n-R}
$$

(B) $m_{1}(R>N) \leq C_{2} \rho_{2}^{N}$. 
Let $\|l\|_{\gamma}$ denote the norm of $l$ as the element of $\left(C^{\gamma}(X)\right)^{*}$.

Corollary 6.2. $\exists C_{3}>0, \rho_{3}<1$ such that $\forall n>0 \forall l_{1}, l_{2} \in E(0)$ $\left\|\mathcal{T}^{n}\left(l_{1}-l_{2}\right)\right\|_{\gamma} \leq C_{3} \rho_{3}^{n}$.

Proof. It suffices to verify this for $l_{j} \in E_{1}(0)$. We have

$$
\left(\mathcal{T}^{n} l_{j}\right)(A)=\int_{Y_{j}} A\left(f^{n} x_{j}\right) d m_{j}\left(x_{j}, t_{j}\right) .
$$

Let $\left(x_{2}, t_{2}\right)=\tau\left(x_{1}, t_{1}\right)$. Then

$$
\left|\mathcal{T}^{n}\left(l_{1}-l_{2}\right)(A)\right| \leq \int_{Y_{1}}\left|A\left(f^{n} x_{1}\right)-A\left(f^{n} x_{2}\right)\right| d m_{1}\left(x_{1}, t_{1}\right) .
$$

Let $Z(n)=\left\{y: R(y)<\frac{n}{2}\right\}$ then

$$
\begin{gathered}
\left|\mathcal{T}^{n}\left(l_{1}-l_{2}\right)(A)\right| \leq \\
\int_{Z(n)}\left|A\left(f^{n} x_{1}\right)-A\left(f^{n} x_{2}\right)\right| d m_{1}\left(x_{1}, t_{1}\right)+2\|A\|_{0} m_{1}\left(Y_{1} \backslash Z(n)\right) \leq \\
\|A\|_{\gamma}\left(\left(C_{1} \rho_{1}^{\frac{n}{2}}\right)^{\gamma}+2 C_{2} \rho_{2}^{\frac{n}{2}}\right) .
\end{gathered}
$$

Let $\nu$ be some $f$-invariant measure in $E(0)$. Substituting in Corollary $6.2 l_{2}=\nu$ we obtain

Corollary 6.3. $\nu$ is the only $f$-invariant measure $\nu$ in $E(0)$. Moreover $\forall l \in E(0) \forall A \in C^{\gamma}(X), \forall n>0$

$$
\left|\int A\left(f^{n} x\right) d l(x)-\nu(A)\right| \leq C_{3} \rho_{3}^{n}\|A\|_{\gamma}
$$

\section{Coupling Algorithm.}

Here we define $\tau$ and $R$ described in Lemma 6.1. Let $\mathcal{Y}$ be the set of rectangles $Y=V \times I, V \in \mathcal{V}, I \subset[0,1]$. Let $D>2$ be a constant defined below (see (13). Let $\tilde{\mathcal{Y}}$ be the set of rectangles $Y=V \times I$, where the length of $V$ is between $\frac{1}{D}$ and $D$ and $I \subset[0,1]$. We write $f(x, t)=(f x, t)$. For $Y_{1} \in \mathcal{Y}, Y_{2} \in \tilde{\mathcal{Y}}$ such that $\operatorname{mes}\left(Y_{1}\right)=\operatorname{mes}\left(Y_{2}\right)$ we give an algorithm defining $\tau$ and $R$. This algorithm will depend on three positive parameters $K, \lambda$ and $\epsilon$. We require $\lambda$ be so close to 0 that $\lambda<-\lambda_{2}$ (recall (1)) and $e^{-\lambda s}>\theta_{1}$, where $s$ and $\theta_{1}$ are the constants from Theorem 4.1. By this Theorem and Proposition 4.3 if $K$ is large enough then

$$
q_{1}=\max _{V \in \mathcal{V}} \operatorname{mes}(U(V))<1
$$

where $U(V)=\left\{x \in V: \exists n>0, y \in V: d\left(f^{n} x, f^{n} y\right) \leq 2\right.$ and $\left.\left(d f^{n} \mid E_{c}\right)(y) \geq K e^{-\lambda_{n}}\right\}$. Take $K$ so large that (10) is satisfied. Write 
$E_{c s}=E_{s} \oplus E_{c}$. By partial hyperbolicity there is a constant $K^{\prime}$ such that $\forall j>0 \forall x$

$$
\left\|\left(d f^{j} \mid E_{c s}\right)(x)\right\| \leq K^{\prime}\left|\left(d f^{j} \mid E_{c}\right)(x)\right| .
$$

Set $\tilde{K}=\max \left(K K^{\prime}, 1\right)$. Since $\left(d f \mid E_{c}\right)(x)$ is Holder continuous there exists $\delta>0$ such that if $d(x, y)<\delta$ then $\left|\left(d f \mid E_{c s}\right)(y)\right| \leq e^{\frac{\lambda}{2}}|d f| E_{c s}(x) \mid$. Let

$$
\epsilon \leq \frac{\delta}{2 \tilde{K}}
$$

Now $D$ is defined by the requirement that if $V_{1}, V_{2}$ are unstable curves, $V_{1} \in \mathcal{V}, V_{2}$ is the image of $V_{1}$ under center-stable projection and $\forall x \in$ $V_{1} d(x, p x)<\delta$ then

$$
\frac{1}{D} \leq \operatorname{length}\left(V_{2}\right)<D
$$

Our algorithm will work recursively. During the first run we define the map between subsets $P_{j}^{\infty}$ of $Y_{j}$. For points where $\tau$ is not defined we define a stopping time $s(y)$ such that the set $P_{j}^{n}=\{y \in$ $\left.Y_{j}: s(y)=n\right\}$ will be of the form $f^{-n}\left(\bigcup_{k} Y_{j n k}\right), Y_{1 n k} \in \mathcal{Y}, Y_{2 n k} \in$ $\tilde{\mathcal{Y}}$ and $\operatorname{mes}\left(P_{1}^{n}\right)=\operatorname{mes}\left(P_{2}^{n}\right)$. Then we can use our algorithm again to couple $P_{1}^{n}$ to $P_{2}^{n}$. More precisely since $\operatorname{mes}\left(P_{1}^{n}\right)=\operatorname{mes}\left(P_{2}^{n}\right)$ we can chop each $Y_{j n k}$ into several pieces so that the resulting collections $\left\{\bar{Y}_{j n l}\right\}$ satisfy $\bigcup_{k} Y_{j n k}=\bigcup_{l} \bar{Y}_{j n l}$ and $\operatorname{mes}\left(\bar{Y}_{1 n l}\right)=\operatorname{mes}\left(\bar{Y}_{2 n l}\right)$. Let $f^{-n} \bar{Y}_{j n l}=U_{j n l} \times I_{j n l}$. Denote $c_{j n l}=\bigcup_{U_{j n l}} \rho_{V_{j}}(x) d x$. Let $\Delta_{j n l}$ be the map $\Delta_{j n l}(x, t)=\left(f^{n} x, r_{j n l}(t)\right)$ where $r_{j n l}$ is the affine isomorphism between $I_{j n l}$ and $\left[0, c_{j n l}\left|I_{j n l}\right|\right]$. (This rescaling is necessary to make $\Delta$ 's measure preserving.) We now call our algorithm recursively to produce maps $\tau_{n l}: \Delta\left(f^{-n} \bar{Y}_{1 n l}\right) \rightarrow \Delta\left(f^{-n} Y_{2 n l}\right)$ and $R_{n l}: \Delta\left(f^{-n} \bar{Y}_{1 n l}\right) \rightarrow \mathbb{N}$ satisfying the conditions of Lemma 6.1. We when set

$$
\begin{gathered}
\tau(x, t)=\left\{\begin{array}{lll}
\tau_{\text {first run }}(x, t) & \text { if } & (x, t) \in P_{1}^{\infty} \\
\Delta_{2 n l}^{-1} \circ \tau_{n l} \circ \Delta_{1 n l} & \text { if } & (x, t) \in f^{-n} \bar{Y}_{1 n l},
\end{array}\right. \\
R(x, t)=\left\{\begin{array}{lll}
R_{\text {first run }}(x, t) & \text { if } \quad(x, t) \in P_{1}^{\infty} \\
n+R_{n l}\left(\Delta_{1 n l}(x, t)\right) & \text { if } \quad(x, t) \in f^{-n} \bar{Y}_{1 n l} .
\end{array}\right.
\end{gathered}
$$

Let us now describe the first run of our algorithm. By rescaling we may suppose that $Y_{j}=V_{j} \times[0,1]$. By u-convergence there is $n_{0}$ and curves $\bar{V}_{j}$ on distance at least 1 from $\partial\left(f^{n} V_{j}\right)$ such that $V_{1} \in \mathcal{V}, \bar{V}_{2}=p \bar{V}_{1}$ and $\forall x \in \bar{V}_{1} d(x, p x) \leq \epsilon$. (Here $p$ means center-stable holonomy.) Let $\hat{c}_{j}=$ $\int_{f^{-n_{0}} \bar{V}_{j}} \rho_{V_{j}}(x) d x$. Let $\left(\bar{t}_{1}, \bar{t}_{2}\right)=\left(1, \frac{\hat{c}_{2}}{\hat{c}_{1}}\right)$ if $\hat{c}_{2} \leq \hat{c}_{1}$ and $\left(\bar{t}_{1}, \bar{t}_{2}\right)=\left(\frac{\hat{c}_{1}}{\hat{c}_{2}}, 1\right)$ if $\hat{c}_{1} \leq \hat{c}_{2}$. Define $\bar{Y}_{j}=\bar{V}_{j} \times\left[0, \bar{t}_{j}\right]$. Let $s(y)=n_{0}$ for points of $Y_{j} \backslash f^{-n_{0}} \bar{Y}_{j}$. 
We now proceed to define $P_{j}^{n}$ inductively for $n>n_{0}$. Let $Q_{j}^{n-1}=$ $Y_{j} \backslash \bigcup_{m=n_{0}}^{n-1} P_{j}^{m}$. We assume by induction that $f^{n-1} Q_{j}^{n-1}=\bigcup_{k} Z_{j k(n-1)}$ where $Z_{j k(n-1)}=V_{j k(n-1)} \times\left[0, t_{j k(n-1)}\right]$,

$$
\operatorname{mes}\left(f^{-(n-1)} Z_{1 k(n-1)}\right)=\operatorname{mes}\left(f^{-(n-1)} Z_{2 k(n-1)}\right),
$$

$V_{1 k(n-1)} \in \mathcal{V}$ and $V_{2 k(n-1)}=p\left(V_{1 k(n-1)}\right)$ and $d(x, p x) \leq r_{n-1}$ where

$$
r_{n}=\tilde{K} \epsilon e^{-\frac{\lambda n}{2}}
$$

Take a Markov decomposition $f V_{1 k(n-1)}=\bigcup_{l} V_{1 l k n}$ and let $V_{2 l k n}=$ $p\left(V_{1 l k n}\right)$. We note that the fact that $V_{1 l k n} \in \mathcal{V},(12)$, (13) and(15) guarantee that then $V_{2 l k n} \in \tilde{\mathcal{Y}}$. Let $\beta_{l k n}=\max _{x \in f^{-n} V_{1 l k n}}\left(d f^{n} \mid E_{c}\right)(x)$. If $\beta_{l k n}>K e^{-\lambda n}$ let $s(y)=n$ on $f^{-n} V_{j l k n} \times\left[0, t_{j k(n-1)}\right]$. Otherwise let $\tilde{Z}_{j l k n}=V_{j l k n} \times\left[0, t_{j k(n-1)}\right]$. In general mes $\left(f^{-n} \tilde{Z}_{1 l k n}\right) \neq \operatorname{mes}\left(f^{-n} \tilde{Z}_{2 l k n}\right)$. So cutoff the top of the larger rectangle so that the adjusted ones satisfy $\operatorname{mes}\left(f^{-n} Z_{1 l k n}\right)=\operatorname{mes}\left(f^{-n} Z_{2 l k n}\right)$ and let $s(y)=n$ on $\tilde{Z}_{j l k n} \backslash Z_{j l k n}$. Now $P_{j}^{\infty}=Y_{j} \backslash \bigcup_{n} P_{1}^{n}$ is a union of vertical intervals of the form $\{(x,[0, t(x)])\}$ where $x$ varies over some positive measure Cantor set $R_{1}$. For points of $P_{1}^{\infty}$ let $\tau(x, t)=\left(p x, \frac{t(p x)}{t(x)} t\right), R(x, t)=n_{0}$.

Four things have to be proven:

$-\tau$ is defined on a set of whole measure in $Y_{1}$;

$-\tau$ is measure preserving;

$-\tau$ satisfies condition (A) of Lemma 6.1;

$-\tau$ satisfies condition (B) of Lemma 6.1.

The second and the third properties of $\tau$ are verified in Section 8 . The first and the fourth properties are verified in Section 9.

\section{Convergence of images.}

Here we prove the property (A) of Lemma 6.1. Let $W_{r}^{*}(x)$ denote the ball centered at $x$ of radius $r$ inside $W^{*}$ with induced Rimannian metric. Let $(x, t) \in P_{1}^{\infty}$. Let $x_{0}=f^{n_{0}} x$. Then $\forall j \geq 0\left(d f^{j} \mid E_{c}\right)\left(x_{0}\right) \leq K e^{-\lambda j}$, so it suffices to show the following.

Lemma 8.1. (Cf. [2], Lemma 2.7) If $x_{0} \in X$ and $n>0$ are such that $\forall 0 \leq j \leq n\left(d f^{j} \mid E_{c}\right)\left(x_{0}\right) \leq K e^{-\lambda j}$ then $\forall 0 \leq j \leq n$

$$
f^{j} W_{\epsilon}^{c s}\left(x_{0}\right) \subset W_{r_{j}}\left(f^{j} x_{0}\right)
$$

where $r_{j}$ is given by (15).

Proof. We proceed by induction. For $j=0$ (16) is true by (12). Suppose that (16) holds for $0 \leq j<j_{0}$. Then $\forall y \in W_{\epsilon}^{c s}(x) \forall 0 \leq j<j_{0}$ 
$d\left(f^{j} y, f^{j} x_{0}\right) \leq \delta$. Hence

$\left|\left(d f^{j_{0}} \mid E_{c s}\right)\right|(y)=\prod_{j=0}^{j_{0}-1}\left|\left(d f \mid E_{c}\right)\left(f^{j} y\right)\right| \leq \prod_{j=0}^{j_{0}-1}\left[e^{\frac{\lambda}{2}}\left|\left(d f \mid E_{c}\right)\left(f^{j} x_{0}\right)\right|\right] \leq K e^{-\frac{j_{0} \lambda}{2}}$.

By $(11)\left\|\left(d f^{j} \mid E_{c s}\right)(y)\right\| \leq \tilde{K} e^{-\frac{j_{0} \lambda}{2}}$ and so $f^{j} W_{\varepsilon}^{c s}\left(x_{0}\right) \subset W_{r_{j}}\left(f^{j} x_{0}\right)$ as claimed.

Note that the proofs of Lemma 8.1 and Theorem 4.1 do not use uconvergence. Hence we get the following result which will be used in Section 10.

Corollary 8.2. Assume that $f$ satisfies all the conditions of Theorem $I$ except possibly $u$-convergence. There are constants $q_{1}, \epsilon>0$ such that for any pair of unstable curves $V_{1}, V_{2}$ such that $V_{1} \in \mathcal{V}, V_{2}=p\left(V_{1}\right)$ and $\forall x \in V d(x, p x)<\epsilon$

$$
\operatorname{mes}\left(\left\{x \in V_{1}: d\left(f^{n} x, f^{n} p x\right) \rightarrow 0\right\}\right) \geq q_{1} .
$$

We now continue with the proof of Lemma 6.1.

Corollary 8.3. $\tau$ is measure preserving.

Proof. By the recursive structure of our algorithm it suffices to show that $\tau: P_{1}^{\infty} \rightarrow P_{2}^{\infty}$ is measure preserving. Denote by $R_{j}$ the base of $P_{j}^{\infty}$. It follows from Lemma 8.1 by standard Pesin theory (see [24], Section 3 or [27], Section 4) that $p: R_{1} \rightarrow R_{2}$ is absolutely continuous. We want to compute its Jacobian $J(x)$. Let $t_{n}(x)$ denote the height of the rectangle containing $(x, 0)$ and $W_{n}(x)$ denote its base. By absolute continuity for almost all $x \in R_{1} x$ is a density point of $R_{1}$ and $p x$ is a density point of $R_{2}$. For such points

$$
J(x)=\lim _{n \rightarrow \infty} \frac{\int_{p W_{n}(x)} 1_{R_{2}}(y) \rho_{V_{2}}(y) d y}{\int_{W_{n}(x)} 1_{R_{1}}(y) \rho_{V_{1}}(y) d y}
$$

Since $R_{1}, R_{2}$ have large densities in $W_{n}(x)$ and $p W_{n}(x)$ respectively we can drop indicator functions. So

$$
J(x)=\lim _{n \rightarrow \infty} \frac{\int_{p W_{n}(x)} \rho_{V_{2}}(y) d y}{\int_{W_{n}(x)} \rho_{V_{1}}(y) d y}=\lim _{n \rightarrow \infty} \frac{t_{n}(x)}{t_{n}(p x)}=\frac{t(x)}{t(p x)}
$$

where the second equality follows by (14). Thus $\tau$ is measure preserving. 


\section{Coupling time.}

Here we prove the part (B) of Lemma 6.1. We begin with some information about one run of our algorithm.

Lemma 9.1. There are constants $q, C_{0}>0, \rho_{0}<1$ such that for any pair $Y_{1}, Y_{2}$

$$
\begin{aligned}
& \text { (1) } \frac{\operatorname{mes}\left(P_{1}^{\infty}\right)}{\operatorname{mes}\left(Y_{1}\right)} \geq q ; \\
& \text { (2) } \frac{\operatorname{mes}\left(P_{1}^{n}\right)}{\operatorname{mes}\left(Y_{1}\right)} \leq C_{0} \rho_{0}^{n} .
\end{aligned}
$$

Proof. We begin with $(2)$. $(y, t)$ can belong to $P_{1}^{n}$ for two reasons. The first, $\exists \tilde{x}$ such that $d\left(f^{n} y, f^{n} \tilde{x}\right) \leq 2$ and $\left(d f^{n} \mid E_{c}\right)(\tilde{x})>K e^{-\lambda n}$. By Proposition $4.3\left(d f^{n} \mid E_{c}\right)(\tilde{x})>K^{*} e^{-\lambda n}$. So the measure of such points is exponentially small by Theorem 4.1 and our choice of $\lambda$. The second reason is that $f^{n}(y, t) \in \tilde{Z}_{1 l k n} \backslash Z_{1 l k n}$. Let

$$
\kappa_{n}(y)=\frac{\operatorname{mes}\left(f^{-n} \tilde{Z}_{1 l k n} \backslash Z_{1 l k n}\right)}{\operatorname{mes}\left(f^{-n} Z_{1 k l n}\right)}=\frac{\operatorname{mes}\left(\tilde{Z}_{1 l k n} \backslash Z_{1 l k n}\right)}{\operatorname{mes}\left(Z_{1 k l n}\right)} .
$$

Lemma 9.2. There are constants $C_{4}>0, \rho_{4}<1$ such that

$$
\kappa_{n}(y) \leq C_{4} \rho_{4}^{n}
$$

Proof. We may suppose that $\operatorname{mes}\left(\tilde{Z}_{1 l k n}\right)>\operatorname{mes}\left(\tilde{Z}_{2 l k n}\right)$, since otherwise $Z_{1 k l n}=\tilde{Z}_{1 k l n}$. Then

$$
\frac{\operatorname{mes}\left(\tilde{Z}_{1 l k n} \backslash Z_{1 l k n}\right)}{\operatorname{mes}\left(Z_{1 k l n}\right)}=\frac{\operatorname{mes}\left(V_{1 l k n}\right) \operatorname{mes}\left(V_{2 k(n-1)}\right)}{\operatorname{mes}\left(V_{2 l k n}\right) \operatorname{mes}\left(V_{1 k(n-1)}\right)}-1 .
$$

Now by inductive assumption we have both on $V_{1 k(n-1)}$ and on $V_{1 l k n}$

$$
d(x, p x) \leq \tilde{K} \varepsilon e^{-\frac{\lambda}{2}(n-1)} .
$$

In the proof below $C_{*}$ will denote various constants which depend on $f$ but not on $n$ or $Y_{j}$. Likewise $\rho_{*}$ will denote various constants which are less then 1. Let $x_{0}$ be the center of $V_{1 k(n-1)}$ and $\tilde{x}_{0}=p x_{0}$. By Holder continuity of unstable foliation $\exists C_{5}>0, \rho_{5}<1$ such that

$$
\left|\frac{\rho\left(x_{0}, x\right)}{\rho\left(\tilde{x}_{0}, p x\right)}-1\right| \leq C_{5} \rho_{5}^{n}
$$

Divide $V_{1 k(n-1)}$ into subintervals $\sigma_{m}$ of size $e^{-\frac{\lambda n}{4}}$ and let $\tilde{\sigma}_{m}=p \sigma_{m}$. Then

$$
\int_{V_{1 k(n-1)}} \rho\left(x_{0}, x\right) d x=\sum_{m} \rho\left(x_{0}, x_{m}\right) d_{m}+O\left(\rho_{6}^{n}\right),
$$


where $x_{m}$ is any point on $\sigma_{m}$ and $d_{m}$ is the distance between the endpoints of $\sigma_{m}$. Likewise

$$
\int_{V_{1 k(n-1)}} \rho\left(x_{0}, x\right) d x=\sum_{m} \rho\left(\tilde{x}_{0}, p x_{m}\right) \tilde{d}_{m}+O\left(\rho_{6}^{n}\right)
$$

where $\tilde{d}_{m}$ is the distance between the endpoints of $\tilde{\sigma}_{m}$. Now by (19) and the triangle inequality $\left|\frac{d_{m}}{\tilde{d}_{m}}-1\right| \leq C_{6} e^{-\frac{\tilde{\lambda} n}{2}}$. Hence

$$
\left|\frac{\operatorname{mes}\left(V_{1 k(n-1)}\right)}{\operatorname{mes}\left(V_{2 k(n-1)}\right)}-1\right| \leq C_{7} \rho_{7}^{n}
$$

Similarly

$$
\left|\frac{\operatorname{mes}\left(V_{1 k l n}\right)}{\operatorname{mes}\left(V_{2 k l n}\right)}-1\right| \leq C_{7} \rho_{7}^{n} .
$$

The last two inequalities together with (18) prove the lemma.

Assertion (2) of Lemma 9.1 now follows from (17) by summation over $k$ and $l$.

Now let $(x, t) \in P_{1}^{\infty}$. Let $\kappa_{j}(x)$ be the relative measure of points cut off the top of the rectangle containing $(x, t)$ on the $j$ th step. Thus $t(x)=\prod_{j=n_{0}}^{\infty}\left(1-\kappa_{j}(x)\right)$. By (17) this series converges uniformly, hence $t(x)$ is uniformly bounded from below. But the measure of the base of $P_{1}^{\infty}$ is also uniformly bounded (see (10)). This proves assertion (1) of Lemma 9.1.

Now represent $R(y)=\sum_{j=1}^{k(y)} s_{j}(y)$, where $s_{j}(x)$ is the stopping time of the $j$ th run of our algorithm. Let $T_{k}$ be the set where $\tau$ is not defined after $k$ runs of our algorithm, $U_{k}=T_{k-1} \backslash T_{k}$. Denote $S_{k}(x)=\sum_{j=1}^{k} s_{j}(x)$ and consider generating functions $\varphi_{k}\left(Y_{1}, \xi\right)=\frac{1}{\operatorname{mes}\left(Y_{1}\right)} \int_{T_{k}} \xi^{S_{k}}(x) d m(y)$, $\psi_{k}\left(Y_{1}, \xi\right)=\frac{1}{\operatorname{mes}\left(Y_{1}\right)} \int_{U_{k}} \xi^{S_{k}}(x) d m(y)$. Lemma 9.1 says that the radius of convergence of $\varphi_{1}$ is strictly greater than 1 and $\varphi_{1}(1) \leq 1-q$. We need the following generalization.

Lemma 9.3. $\exists \delta_{0}, \bar{q}, C>0$ such that if $0 \leq \xi \leq 1+\delta_{0}$ then

(1) $\varphi_{k+1}(\xi) \leq(1-\bar{q}) \varphi_{k}(\xi)$

(2) $\psi_{k+1} \leq \bar{C} \varphi_{k}(\xi)$.

Proof. (1) Take some $y \in T_{k}$. Assume that after $k$ runs of our algorithm $f^{S_{k}(y)} \in Y_{1}^{k}(y)$ and $(k+1)$-st run couples $Y_{1}^{k}$ to some $Y_{2}^{k}$. Let us compare the contributions of $Y_{1}^{k}$ to $\varphi_{k}$ and $\varphi_{k+1}$. (That is if $Y_{1}^{k}=\Delta^{(k)} \bar{Y}_{1}^{k}$ where $\bar{Y}_{1}^{k} \subset Y_{1}, \Delta^{(k)}(x, t)=\left(f^{S_{k}(y)} x, a t+b\right)$ and we compare

$$
I_{k}=\frac{1}{\operatorname{mes}\left(Y_{1}\right)} \int_{\bar{Y}_{1}^{k}} \xi^{S_{k}(x)} d m_{1}=\xi^{S_{k}(y)} \frac{\operatorname{mes}\left(\bar{Y}_{1}^{k}\right)}{\operatorname{mes}\left(Y_{1}\right)}
$$


and $I_{k+1}=\frac{1}{\operatorname{mes}\left(Y_{1}\right)} \int_{\bar{Y}_{1}^{k}} \xi^{S_{k+1}(x)} d m_{1}$.) Their ratio equals $r(\xi)=\frac{I_{k+1}}{I_{k}}=$ $\varphi_{1}\left(Y_{1}^{k}, \xi\right)$. By Lemma $9.1 r(\xi)$ is uniformly convergent and $r(1) \leq 1-q$. So there is $\delta_{0}, \bar{q}$ such that for $\xi<1+\delta_{0} r(\xi) \leq 1-\bar{q}$. This proves (1).

(2) $\psi_{k+1}(\xi)=\xi^{n_{0}} \int_{U_{k+1}} \xi^{S_{k}(y)} d m(y) \leq \xi^{n_{0}} \int_{T_{k}} \xi^{S_{k}(y)} d m(y) \leq\left(1+\delta_{0}\right)^{n_{0}} \varphi_{k}(\xi)$.

Now for $\xi \leq 1+\delta_{0}$

$$
\int_{Y_{1}} \xi^{R(y)} d m(y)=\sum_{k=1}^{\infty} \psi_{k}(\xi) \leq C \sum_{k=1}^{\infty}(1-\bar{q})^{k-1} \varphi_{1}(\xi) \leq \frac{C}{\bar{q}} \varphi_{1}(\xi)<\infty .
$$

This shows that $m(R>n) \leq \frac{\text { Const }}{\left(1+\delta_{0}\right)^{n}}$ and in particular $m(R=\infty)=0$. These facts complete the proof of Lemma 6.1.

\section{Proof of the MAIN Results.}

Proof of Theorem I. Consider $l \in E(R)$. By Proposition 5.1 there exists $\tilde{l} \in E(0)$ such that for all $N>0$

$$
\left\|\mathcal{T}^{N+\frac{n}{2}} l-\mathcal{T}^{N} \tilde{l}\right\| \leq \text { Conste } \frac{\frac{-\lambda_{5} n}{2}}{} .
$$

Hence

$$
\left\|\mathcal{T}^{n} l-\nu\right\| \leq \text { Const } e^{\frac{-\lambda_{5} n}{2}}+\left\|\mathcal{T}^{\frac{n}{2}} \tilde{l}-\nu\right\| \leq C_{8} \rho_{8}^{n}
$$

It follows from [12] that $\nu$ is a global attractor for $f$. Let $B \in C^{\gamma}(X)$ then, for large $R, B$.Lebesgue and $B \cdot \nu$ are in $E(R)$, provided that $\gamma$ is sufficiently close to 1 (see $[26,12]$ ). This together with Proposition 5.2 proves Theorem I for $\gamma$ close to 1 . The result for general $\gamma$ is proved by approximation of $A$ and $B$ by smooth functions.

Proof of TheOREM II. By the remark at the end of Section 3 there exists a $C^{2}$ neighborhood $\mathcal{O}_{1}(f)$ such that any $g \in \mathcal{O}_{1}(f)$ is mostly contracting. Also, given $\varepsilon$ there is another $C^{2}$ neighborhood $\mathcal{O}_{2}(f)$ such that $\forall g \in \mathcal{O}_{2}(f) \exists n_{0}$ such that $\forall V_{1}, V_{2} \in \mathcal{V} \exists U_{j} \subset V_{j}$ such that $g^{n_{0}} U_{1} \in \mathcal{V}, U_{2}=p U_{1}$ and $\forall x \in U_{1} d\left(g^{n_{0}} x, g^{n_{0}} p x\right)<\varepsilon$. By Corollary 8.2 $g$ is $\mathrm{u}$-convergent. Constants $C, \zeta$ from part (b) of Theorem I can be chosen uniformly for $g$ near $f$ since they depend only on Holder data of invariant foliations and the constant $\alpha_{0}$ in (6). Thus

$$
\begin{gathered}
\nu_{g}(A)-\nu_{f}(A)=\int\left[A\left(g^{n} x\right)-A\left(f^{n} x\right)\right] d x+O\left(\|A\|_{\gamma} \zeta^{n}\right)= \\
\|A\|_{\gamma}\left(O\left(\zeta^{n}\right)+O\left(\left(K^{n} d(f, g)\right)^{\gamma}+\zeta^{n}\right)=\right. \\
\left.\|A\|_{\gamma} O\left(K^{n} d(f, g)\right)^{\gamma}+\zeta^{n}\right) .
\end{gathered}
$$

Taking $n$ so that $\left(\frac{\zeta}{K^{\gamma}}\right)^{n}=d(f, g)^{\frac{\gamma}{2}}$ we obtain the result needed. 


\section{EXAMPLES OF U-CONVERGENT DIFFEOMORPHISMS.}

Here we give several conditions sufficient for u-convergence.

(a) Suppose that $f$ has 3-leg accessibility property in the sense that there exists $R$ such that $\forall V_{1}, V_{2} \in \mathcal{V} \exists x_{1} \in V_{1}, x_{2} \in V_{2}$ such that $x_{2} \in W^{s}\left(x_{1}\right)$ and $d_{s}\left(x_{1}, x_{2}\right) \leq R$ where $d_{s}$ means the distance along the stable leaf. Then $d_{s}\left(f^{n} x_{1}, f^{n} x_{2}\right) \leq e^{\lambda_{2} n} R$ so $f$ is u-convergent.

(b) Assume that $W^{u}$ is minimal. Thus given $\varepsilon$ there exists $R$ such that for any two unstable curves $V_{1}, V_{2}$ of length at least $R$ there are $x_{j} \in V_{j}$ such that $d\left(x_{1}, x_{2}\right) \leq \varepsilon$. So, $f$ is u-convergent.

(c) To formulate this condition suppose that the fibers of $W^{c}$ are circles. Suppose that $f$ satisfies the following condition: for any two unstable curves $V_{1} \in \mathcal{V}$ and $V_{2} \in W^{c u}\left(V_{1}\right): V_{2}=p_{c}\left(V_{1}\right)$ (where $p_{c}$ denotes the center holonomy) the following inequality holds

$$
\int_{V_{1}} \rho_{V_{1}}(x) \ln \left(\frac{d(f x, f p x)}{d(x, p x)}\right) d x \leq-\alpha_{0}<0 .
$$

(Note that letting here $V_{2}$ tend to $V_{1}$ we obtain (6) so (20) is a stronger assumption).

Proposition 11.1. there are sets $U_{j} \subset V_{j}$ such that $\operatorname{mes}\left(V_{j} \backslash U_{j}\right)=0$, $U_{2}=p\left(U_{1}\right)$ and for all $x \in U_{1} d\left(f^{n} x, f^{n} p x\right) \rightarrow 0$.

Proof. Let $U_{1}=\left\{x \in V_{1}: d\left(f^{n} x, f^{n} p x\right) \rightarrow 0\right\}$. Repeating the arguments of Corollary 8.2 we get that there is a constant $\tilde{q}$ (depending only on $f$ but not on $V_{1}, V_{2}$ ) such that

$$
\operatorname{mes}\left(U_{1}\right) \geq \tilde{q} \operatorname{mes}\left(V_{1}\right) .
$$

Now considering Markov decompositions $f^{n} V_{1}=\bigcup_{l} V_{l n}$ and applying (21) to each $V_{l n}$ we find that $V_{1} \backslash U_{1}$ has no density points and so $\operatorname{mes}\left(V_{1} \backslash U_{1}\right)=0$. Interchanging $V_{1}$ and $V_{2}$ we get $\operatorname{mes}\left(V_{2} \backslash U_{2}\right)=0$.

Now since $W^{u}$ and $W^{c s}$ are transverse foliations there exists $R>0$ such that $\forall x_{1}, x_{2} \in X$ there is $x_{3} \in X$ such that $x_{3} \in W^{c s}\left(x_{1}\right) \cup W^{u}\left(x_{2}\right)$ and $d_{c s}\left(x_{1}, x_{3}\right)<R, d_{u}\left(x_{1}, x_{3}\right)<R$. Iterating this construction forward we find that $\forall \delta \exists \tilde{R}$ such that $\forall x_{1}, x_{2} \exists y_{1}, y_{2}$ such that $y_{1} \in W_{\delta}^{s}\left(x_{1}\right)$ and $y_{2} \in W^{c}\left(y_{1}\right) \bigcap W_{\tilde{R}}^{u}\left(x_{2}\right)$. Now if $\delta$ is small enough then by Corollary 8.2 there exist sets $U_{1} \subset W_{1}^{u}\left(U_{1}\right), \tilde{U} \subset W_{1}^{u}\left(y_{1}\right)$ such that $\operatorname{mes}\left(U_{1}\right)>0$, $\operatorname{mes}\left(\tilde{U}_{1}\right)>0 \tilde{U}_{1}=p_{c s}\left(U_{1}\right)$ and $\forall x \in U_{1} d\left(f^{n} x, f^{n} p_{c s} x\right) \rightarrow 0$. But $y_{2} \in W_{\tilde{R}}^{u}\left(x_{2}\right)$. By compactness there is $R^{*}>0$ such that $U_{2} \subset W_{R^{*}}\left(x_{2}\right)$. Thus $\exists z_{1} \in W_{1}^{u}\left(x_{1}\right), z_{2} \in W_{R^{*}}^{u}\left(x_{2}\right)$ such that $d\left(f^{n} z_{1}, f^{n} z_{2}\right) \rightarrow 0$. Now take $V_{1}, V_{2} \in \mathcal{V}$. There exists $n_{0}$ such that the lengths of $f^{n_{0}} V_{j}$ is greater than $2 R^{*}$ and so $\exists z_{j} \in f^{n_{0}} V_{j}$ : such that $d\left(f^{n} z_{1}, f^{n} z_{2}\right) \rightarrow 0$. Therefore 
$f$ is $\mathrm{u}$-convergent. Hence (20) implies both mostly contractiveness and $\mathrm{u}$-convergence.

Similarly we can consider the case when the leaves of $f$ are noncompact and require that (20) is satisfied for $d\left(V_{1}, V_{2}\right) \leq R$ there $R=$ $R(f)$ is a large constant.

Remark. If fibers of $W^{c}$ are circles and (20) is satisfied then one can show using Proposition 11.1 that $W^{c}$-holonomy restricted to $W^{c u}$ is absolutely continuous. Let us examine underlying geometric picture more closely since it will allow the reader to appreciate better the idea behind the proof of Lemma 6.1. Indeed choose an orientation of $W^{c}$ and let $V_{1}, V_{2}$ be two unstable curves in the same center-unstable leaves such that $V_{2}=p\left(V_{1}\right) V_{2}$ is $\varepsilon$-close to $V_{1}$ and is on the right of $V_{1}$. Then there are subsets $U_{j} \subset V_{j}$ such that $U_{2}=p\left(U_{1}\right), \operatorname{mes}\left(V_{j} /\right.$ $\left.U_{j}\right)=0$ and $\forall x \in U_{1} d\left(f^{n} x, f^{n} p x\right) \rightarrow 0$. The geometry of $U_{1}$ is however quite complicated. In fact there is a Cantor set $U_{1}^{(1)} \subset U_{1}$ of measure $1-O\left(\varepsilon^{C}\right)$ such that for $x \in U_{1}^{(1)} f^{n} p x$ is always on the right from $f^{n} x$. Each gap of $U_{1}^{(1)}$ contains a positive measure Cantor set $U_{1}^{(2)}$ such that for $x \in U_{1}^{(2)} f^{n}(p x)$ is on the left from $f^{n} x$. In turn each gap of $U_{1}^{(2)}$ contains a positive measure Cantor set $U_{1}^{(3)}$ such that for $x \in U_{1}^{(2)}$ $f^{n}(p x)$ is on the right from $f^{n} x$ and so on.

If fibers of $W^{c}$ are lines then it is conceivable that $W^{c}$ is not absolutely continuous inside $W^{c u}$. Instead Lemma 6.1 allows us to construct a map $\pi: V_{1} \rightarrow V_{2}$ which is absolutely continuous and such that $\pi x \in W^{c s}(x)$. However even if $V_{2}$ is very close to $V_{1}$ still $\pi x$ sometimes will be different from the naive projection along the $W^{c}$-fibers. The fact that the fibers of $W^{c s}$ are dense and so for each $x$ there is a countable number of candidates for $\pi x$ is really essential to this proof.

\section{EXAMPlES OF MOSTLY CONTRACTING DIFFEOMORPHiSMS.}

(a) Let $T: \mathbb{T}^{2} \rightarrow \mathbb{T}^{2}$ be a linear Anosov diffeomorphism. Take $A: \mathbb{T}^{2} \rightarrow S L_{2}(\mathbb{R})$. Assume that the image $A\left(\mathbb{T}^{2}\right)$ generates $S L_{2}(\mathbb{R})$.

Let $S_{n_{1}, n_{2}}(x)=A\left(T^{n_{1} n_{2}} x\right) \ldots A\left(T^{n_{2}} x\right) A(x), M_{n_{1}, n_{2}}(x) v=\frac{S_{n_{1}, n_{2}}(x) v}{\left\|S_{n_{1}, n_{2}}(x) v\right\|}$. Define $f_{n_{1}, n_{2}}: \mathbb{T}^{2} \times \mathbb{P}^{1} \rightarrow \mathbb{T}^{2} \times \mathbb{P}^{1}$ by $f_{n_{1} n_{2}}(x, v)=\left(T^{n_{1} n_{2}} x, M_{n_{1}, n_{2}} v\right)$. We claim that $\exists \bar{n}_{2}$ such that $\forall n_{2}>\bar{n}_{2} \exists \bar{n}_{1}$ such that $\forall n_{1}>\bar{n}_{1} f_{n_{1}, n_{2}}$ satisfy $(20)$. Let $d\left(v_{1}, v_{2}\right)=\operatorname{Area}\left(v_{1}, v_{2}\right)$. We have

$$
\begin{gathered}
d\left(f_{n_{1}, n_{2}}\left(x, v_{1}\right), f_{n_{1}, n_{2}}\left(x, v_{2}\right)\right)= \\
\operatorname{Area}\left(M_{n_{1}, n_{2}}(x) v_{1}, M_{n_{1}, n_{2}}(x) v_{2}\right)= \\
\frac{\operatorname{Area}\left(S_{n_{1}, n_{2}}(x) v_{1}, S_{n_{1}, n_{2}}(x) v_{2}\right)}{\left.\left\|S_{n_{1}, n_{2}}(x) v_{1}\right\| \| S_{n_{1}, n_{2}}(x) v_{2}\right) \|}=
\end{gathered}
$$




$$
\frac{\operatorname{Area}\left(v_{1}, v_{2}\right)}{\left.\| S_{n_{1}, n_{2}}(x) v_{1}|||| S_{n_{1}, n_{2}}(x) v_{2}\right) \|}
$$

since $S \in S L_{2}(\mathbb{R})$. The reader will have no difficulties to show that for fixed $n_{2}$ and large $n_{1} f_{n_{1}, n_{2}}$ is partially hyperbolic, its unstable manifolds are graphs of functions $\Gamma_{n}: W^{u}\left(x_{0}\right) \rightarrow \mathbb{P}^{1}$ and $\left\|d \Gamma_{n}\right\| \rightarrow 0$ as $n \rightarrow$ $\infty$. Now the Riemann structure of $\mathbb{T}^{2}$ is non-degenerate on the leaves of $W^{u}$ and with respect to this structure $\rho\left(\left(x_{1}, \Gamma_{n}\left(x_{1}\right)\right),\left(x_{2}, \Gamma_{n}\left(x_{2}\right)\right)\right)=$ $\rho\left(x_{1}, x_{2}\right)$. Let $m$ be the distribution of $A(x)$ with respect to Lebesgue measure and $m_{n}$ be the $n$-th convolution power of $m$. Take now $x_{0} \in \mathbb{T}^{2}$, $v_{1}, v_{2} \in \mathbb{P}^{1}$ and let $\Gamma_{n}^{j}(x)$ be the function defining the unstable manifold through $\left(x_{0}, v_{j}\right)$. Then

$$
\begin{gathered}
\int_{V} \rho(z) \ln \left(\frac{d\left(f_{n_{1}, n_{2}}\left(x, \Gamma_{n}^{1}(x)\right), f_{n_{1}, n_{2}}\left(x, \Gamma_{n}^{2}(x)\right)\right)}{d\left(v_{1}, v_{2}\right)}\right) d z \rightarrow \\
-\frac{1}{|V|} \int_{V} \mathbb{E}_{m_{n_{2}-1}}\left(\ln \left\|A M(x) \Gamma_{n}^{1}(x)\right\|+\ln \left\|A M(x) \Gamma_{n}^{2}(x)\right\|\right) d x
\end{gathered}
$$

as $n_{1} \rightarrow \infty$. But by [5], Theorem A3.6 for all $v \frac{1}{n} \mathbb{E}_{m_{n}} \ln \|A v\| \rightarrow \lambda_{+}>0$ where $\lambda_{+}$is the positive exponent of $m$. So for large $n_{2}, n_{1} \gg n_{2}$ the expression (22) is negative.

(b) This example is similar to (a). Let $f_{n}: \mathbb{T}^{2} \times S^{1} \rightarrow \mathbb{T}^{2} \times S^{1}$ be given by $f_{n}(x, y)=\left(T^{n} x, g_{n}(x) y\right)$ where the distribution of $g_{n}$ converges to that of time 1 map of the stochastic differential equation

$$
d y=\Phi(y) d w(t)
$$

$d w(t)$ being the white noise. Then for large $n f_{n}$ is mostly contracting. This follows from the fact that $\xi=\frac{\partial y}{\partial y_{0}}(t)$ satisfies

$$
d \ln \xi=\frac{d \Phi}{d y} d w-\frac{1}{2}\left(\frac{d \Phi}{d y}\right)^{2} d t
$$

and so

$$
\mathbb{E}_{\mathbf{P}} \ln \xi(1)=-\frac{1}{2} \int_{0}^{1} \mathbb{E}_{\mathbf{P}}\left(\frac{d \Phi}{d y}(t)\right) d t<0 .
$$

where $\mathbf{P}$ denotes the stationary distribution of process defined by (23). (Of course since the distribution of $\xi$ is not compactly supported some restrictions should be imposed on the rate of convergence of $g_{n}$ to the distribution of the solution of (23). We leave it as an exercise to the reader to write down the explicit estimates.)

Remark. This example shows that many phenomena occurring in stochastic differential equation can also take place in deterministic systems. More research is needed in this direction. 
(c) The above examples are essentially dissipative, since where both $f$ and $f^{-1}$ are mostly contracting. Here we describe a conservative example which is a slight modification of the one given in [32].

Let $T: \mathbb{T}^{2} \rightarrow \mathbb{T}^{2}$ be as before. Consider $f_{0}: \mathbb{T}^{3} \rightarrow \mathbb{T}^{3}$ given by $f_{0}(x, \theta)=(T(x), \theta+\tau(x))$ where $\tau$ is such that $f_{0}$ is Bernoullian (this condition discards an infinite codimension submanifold in the space of skewing functions). Let $h: \mathbb{T}^{3} \rightarrow \mathbb{T}^{3}$ be a volume preserving diffeomorphisms close to identity. ( $d f$ maps small cones $\mathcal{K}_{u}$ around $E_{u}$ and $\mathcal{K}_{c u}$ around $E_{u} \wedge E_{c}$ into themselves. Likewise $d f^{-1}$ preserves cones $\mathcal{K}_{s}$ and $\mathcal{K}_{c s}$. We want $d h$ map $E_{u}$ into $\mathcal{K}_{u}$ and so on.) Let $f_{n}=f_{0}^{n} \circ h \circ f_{0}^{n}$. Choose vectorfields $e_{u} \in E_{u}, e_{c} \in E_{c}$ and $e_{s} \in E_{s}$ so that $d f\left(e_{u}\right)=\lambda e_{u}$, $d f\left(e_{c}\right)=e_{c}, d f\left(e_{s}\right)=\frac{1}{\lambda} e_{s}$. Suppose that in this basis $d h(x)=\left(A_{i j}(x)\right)$. $f_{n}$ has an unstable vector of the form $v_{u}=e_{u}+\alpha(x) e_{c}+\beta(x) e_{s}$. Let $d f_{n}(x) v_{u}=r_{1}^{(n)}(x) v_{u}$. The direct calculation shows that $r_{1}^{(n)}(x)=$ $\lambda^{2 n} A_{11}\left(f_{0}^{n} x\right)\left(1+O\left(\frac{1}{\lambda^{n}}\right)\right)$. Similarly choose the central vector $v_{0}$ so that $v_{u} \wedge v_{0}=e_{u} \wedge e_{0}+\ldots$ and let $d f_{n}(x)\left(v_{u} \wedge v_{0}\right)=r_{2}^{(n)}(x)\left(v_{u} \wedge v_{0}\right)$. Then $r_{2}^{(n)}(x)=\lambda^{2 n}\left(A_{11} A_{22}-A_{12} A_{21}\right)\left(f_{0}^{n} x\right)\left(1+O\left(\frac{1}{\lambda^{n}}\right)\right)$. Again one can show that unstable manifolds of $f_{n}$ are close to unstable manifolds of $f_{0}$ and so they are transversal to $W^{c s}$. Let

$$
\mathcal{J}(h)=\int_{\mathbb{T}^{3}} \ln \left(\frac{A_{11} A_{22}-A_{12} A_{21}}{A_{11}}(x)\right) d x .
$$

We have

$$
\begin{gathered}
\int_{V_{n}} \rho_{V_{n}}(x) \ln \left(d f_{n} \mid E_{c}\right)(x) d x \approx \\
\int_{V_{n}} \rho_{V_{n}}(x) \ln \left(\frac{A_{11} A_{22}-A_{12} A_{21}}{A_{11}}\right)\left(f_{0}^{n} x\right) d x
\end{gathered}
$$

Since $f_{0}$ is mixing we obtain (cf. [18] or [15] Section 20.6) that

$$
\begin{gathered}
\int_{V_{n}} \rho_{V_{n}}(x) \ln \left(\frac{A_{11} A_{22}-A_{12} A_{21}}{A_{11}}\right)\left(f_{0}^{n} x\right) d x \approx \\
\mathcal{J}(h) \int_{V_{n}} \rho_{V_{n}}(x) d x \approx \mathcal{J}(h) .
\end{gathered}
$$

So, if $\mathcal{J}(h)<0$ then $f_{n}$ is mostly contracting for large $n$. Similarly if $\mathcal{J}(h)>0$ then $f_{n}^{-1}$ is mostly contracting for large $n$. (A more symmetric expression for $\mathcal{J}$ is

$$
\left.\mathcal{J}=\int_{\mathbb{T}^{3}}\left[\ln A_{33}\left(h^{-1}\right)-\ln A_{11}(h)\right] d x .\right)
$$


To show that $\mathcal{J}$ is not identically zero one can use the following argument of [32]. Let $\operatorname{Diff}^{*}\left(\mathbb{T}^{3}\right)$ be the space of volume preserving $C^{3}$ diffeomorphisms which preserve $W^{c s}\left(f_{0}\right)$. Then $\left.\mathcal{J}\right|_{\text {Diff* }\left(\mathbb{T}^{3}\right)}$ is a $C^{2}$-functional and calculating its first two derivatives at identity one can prove that $\mathcal{J} \not \equiv 0$. We refer the reader to [32] for more details.

(d) A similar example can be given with $f_{0}$ being a time one map of the geodesic flow on unit tangent bundle over a negatively curved surface.

(e) In [4] several examples are given of the systems having the following property

(*) $\forall V \in \mathcal{V}$ there is a subset $U \subset V$ of positive measure such that for all $x \in U$ the forward Lyapunov exponent of $E_{c}$ is negative.

The next proposition is essentially proven in [4] even though it is not stated where. For the convenience of the readers we sketch their arguments below.

Proposition 12.1. $f$ satisfies $(*) \Leftrightarrow$ it is mostly contracting.

Proof. In view of Theorem 4.1 we only have to show that if $f$ satisfies $\left(^{*}\right)$ then it is mostly contracting. Given $x_{0} \in X$ choose $V$ containing $x_{0}$ in its interior. By $\left(^{*}\right) \exists K\left(x_{0}\right), \lambda\left(x_{0}\right)$ such that the set $L\left(x_{0}\right)=\{x \in$ $\left.V:\left(d f^{n} \mid E_{c}\right)(x) \leq K e^{-\lambda n}\right\}$ has positive measure. Given $\delta$ there is $\varepsilon>0$ and a positive measure subset $\tilde{L} \subset L$ such that

(a) $\forall x \tilde{L} W_{\varepsilon}^{c s}(x)$ belong to the weak stable manifold of $x$;

(b) If $\tilde{V} \in \mathcal{V}, d(\tilde{V}, V) \leq \varepsilon$ the the center stable holonomy $p: V \rightarrow \tilde{V}$ is absolutely continuous on $\tilde{L}$ and its Jacobian $J(x)$ satisfies $|J(x)-1| \leq$ $\delta$.

Let $y$ be a density point of $\tilde{L}\left(x_{0}\right)$. It follows that if $I$ is small enough interval about $y$ then $\forall n$

$$
\int_{I} \rho_{I}(x) \ln \left(d f^{n} \mid E_{c}\right)(x) d x \leq-\frac{\lambda n}{2}+K_{1} .
$$

Let $C=\left[I, W_{\varepsilon}^{c s}(y)\right]([\cdot, \cdot]$ denotes (u,cs)-local product). Then if $\delta, \varepsilon$ are small enough then for any unstable slice $J$ of $C$

$$
\int_{J} \rho_{J}(x) \ln \left(d f^{n} \mid E_{c}\right)(x) d x \leq-\frac{\lambda n}{4}+K_{2}
$$

Call $T=\left[V\left(x_{0}\right), W_{\varepsilon\left(x_{0}\right)}^{c s}\left(y\left(x_{0}\right)\right)\right]$ the trap associated with $x_{0}$. Call $C$ the core of $T$. By compactness $X$ is covered by a finite number of traps $\left\{T_{j}\right\}$. Now take any $V \in \mathcal{V}$. Given $m>0$ let $V_{1}(m)$ be the set of points which visit some $C_{j}$ before time $m$ and let $V_{2}(m)=V \backslash V_{1}(m)$. We have

$$
\mathcal{I}_{n}=\int_{V} \rho_{V}(x) \ln \left(d f^{n} \mid E_{c}\right)(x) d x=
$$




$$
\begin{gathered}
\left(\int_{V_{1}(m)}+\int_{V_{2}(m)}\right) \rho_{V}(x) \ln \left(d f^{n} \mid E_{c}\right)(x) d x=I+I I \\
I \leq\left[-\frac{\lambda n}{4}+K(m)\right] \operatorname{mes}\left(V_{1}(m)\right) \\
I I \leq \text { Constnmes }\left(V_{2}(m)\right)
\end{gathered}
$$

and $\operatorname{mes}\left(V_{2}(m)\right) \leq$ Const $\theta^{m}$ for some $\theta<1$ (the proof of this last inequality is similar to that of Lemma 9.1.) So, for large $n, \mathcal{I}_{n}$ is negative.

\section{Conclusions.}

Here we repeat what we have said in the introduction adding more technical details.

13.1. Here we relate our results with those of [38]. Let $K, \lambda$ be as in Section 7. For $V \in \mathcal{V}$ let $x(V)$ be the center of $V$ and

$$
L(V)=\left\{x \in V:\left(d f^{n} \mid E_{c}\right)(x) \leq K e^{-\lambda n}\right\} .
$$

Let $\Lambda_{\delta}(V)=\left[L, W_{\delta}^{c s}(x(V))\right]$. Then we have essentially shown that if $\delta$ is small enough then $\Lambda_{\delta}(V)$ satisfies the conditions of Theorem 2 of [38]. We did not deduce our result from [38] but rather repeated some of her arguments in Sections 6-9 in order to show that u-convergence guarantees the absence of the discrete spectrum. Now suppose that $f$ satisfies all the conditions of Theorem I except u-convergence. Then the conclusion can be false (consider, for example, the double covering of $f$ from $12(\mathrm{a})$ corresponding to $\left.\pi: S^{1} \rightarrow \mathbb{P}^{1}\right)$. However we can still say something. Namely by [4] there is a finite number $\nu_{1} \ldots \nu_{k}$ of SRB measures and the union of their basins is the whole of $X$. Let $\Omega=$ $\bigcup_{j} \operatorname{supp}\left(\nu_{j}\right)$. Let $\epsilon$ be as in Section 7 . Choose a finite disjoint set $V_{1} \ldots V_{m}$ which is $\epsilon$-dense in $\Omega$. Take $\delta \ll \epsilon$. Choose small subintervals $U_{j} \subset V_{j}$ such that $\Lambda_{\delta}\left(U_{l}\right)$ are disjoint. Then the arguments of Sections 8 and 9 show that $\Lambda_{\delta}=\bigcup_{l=1}^{m} \Lambda_{\delta}\left(U_{l}\right)$ satisfy Theorem 2 of [38] except maybe $f^{n}$ is not ergodic for some $n$. It then follows from the analysis of [38] that $\forall j \exists n_{j}$ so that $\nu_{j}=\frac{1}{n_{j}} \sum_{l=0}^{n_{j}} \nu_{j l}$ and $\left(f^{n_{j}}, \nu_{j l}\right)$ is exponentially mixing.

Question. What happens for $g$ close to $f$ ? Can the maps $g \rightarrow \nu_{j}(g)$ be chosen continuously? 
13.2. It seems that the assumption that $f$ is dynamically coherent can be relaxed (it is however satisfied in all the known examples). In fact, we only used it in Section 8 . Let $\left(\Omega_{1}, m_{1}\right)$ and $\left(\Omega_{2}, m_{2}\right)$ be probability spaces. Call the map $\tau: \Omega_{1} \rightarrow \Omega_{2} \varepsilon-$ measure preserving if $\exists A_{j} \subset \Omega_{j}$ such that $m_{j}\left(A_{j}\right) \leq \varepsilon$ and $\left.\tau\right|_{\Omega_{1} \backslash A_{1}}$ is absolutely continuous and the Jacobian satisfies $|J(x)-1| \leq \varepsilon$. For our arguments it suffices to know that if $d\left(V_{1}, V_{2}\right) \leq \varepsilon$ then there is $\varepsilon^{\beta}$-measure preserving map $p: V_{1} \rightarrow V_{2}$ such that for $x \in V_{1} \backslash A_{1} f^{n}(x)$ and $f^{n}(p x)$ converge exponentially fast. This, in turn seems to follow from the Pesin theory. However, the proof without the dynamical coherence assumption would be much more complicated.

13.3. Question. Let $f$ be as in Theorem I. Is the map $g \rightarrow \nu_{g}$ actually smooth? An easier problem is the following. Assume that $W^{c}(f)$ is absolutely continuous. Is the map $g \rightarrow \nu_{g}$ differentiable at $f$ ? (See [31] for additional discussion.)

13.4. Question. How common is (6) among partially hyperbolic diffeomorphisms of three manifolds? In particular is the set $\left\{f: f\right.$ or $f^{-1}$ is mostly contracting \} dense?

13.5. Let $X$ be a three dimensional manifold. Consider the space $\mathcal{S}$ of partially hyperbolic ergodic volume preserving diffeomorphisms with two negative Lyapunov exponents.

Question. How often are elements of $\mathcal{S}$ mostly contracting? What is the rate of mixing for elements of $\mathcal{S}$ ?

According to the general scheme proposed in [38] one has to locate a 'bad set' of $f$ and see how long an orbit can stay near it. Analysis of [4] shows that the bad set here is the the set of points whose forward orbits never fall into any trap described in Section 12(e). So a way to attack this problem is to obtain more information about the geometry of this set. For example, can it have the Hausdorff dimension equal to three?

13.6. For $f \in \mathcal{S}$ we have the following elegant characterization due to $[4]$

$f$ is mostly contracting if and only if $W^{u}(f)$ is minimal.

(Minimality implies mostly contractiveness by ([4], Theorem B). The converse implication is easier. See, for example [18].)

Question. How often $W^{u}$ is minimal? What can be said if it is not? 
ON DYNAMICS OF MOSTLY CONTRACTING DIFFEOMORPHISMS.

13.7. Another natural condition to consider if $\operatorname{dim} W^{c}=1$ is

$$
\forall V \in \mathcal{V} \quad \int_{V} \rho_{V}(x) \ln \left(d f \mid E_{c}\right)(x) d x \geq \alpha_{0}>0 .
$$

In this case the results similar to ours were obtained in [1], [2]. In fact, [1], [2], [4] do not assume that $\operatorname{dim}\left(W^{c}\right)=1$ but only that all its Lyapunov exponents have the same sign.

Question. Can a similar theory be developed in case $\left(f \mid E_{u}\right)$ has both positive and negative Lyapunov exponents?

13.8. In the example 12(a) $f$ is a skew extension over Anosov base. Similar construction can be made with Axiom A attractors.

Question. What can be said for general Axiom A diffeomorphisms? In particular, call $f$ entropy stable if any $g$ near $f$ has an unique measure of maximal entropy $\mu_{g}$ and $\mu_{g} \rightarrow \mu_{f}$ as $g \rightarrow f$. How large is the set of entropy stable diffeomorphisms? Are examples of Section 12 entropy stable?

13.9. In $[7,8,16,20,28]$ a number of examples is given of ergodic systems which remain ergodic after a small volume preserving perturbations.

Question. What happens if we allow non-volume preserving perturbations?

13.10. Finally, let us remark that the questions we asked are special cases of some general conjectures about statistical properties of a generic dynamical systems. See [22].

\section{REFERENCES}

[1] Alves J. SRB measures for nonhyperbolic systems with multidimensional expansion IMPA Thesis, 1997.

[2] Alves J., Bonatti C. \& Viana M., SRB measures for partially hyperbolic diffeomorphisms: the expanding case, preprint.

[3] Benedics M. \& Young L.-S. Sinai-Bowen-Ruelle measures for certain Henon maps. Inv. Math. 112 (1993) 541-576.

[4] Bonatti C. \& Viana M. SRB measures for partially hyperbolic systems those central direction is mostly contracting, to appear in Israel Math. J.

[5] Bougerol P. \& Lacroix J. Products of random matrices with applications to Schrodinger operators Birkhauser, Boston-Basel-Stuttgart, 1985.

[6] Brin M. \& Pesin Ya. B. Partially hyperbolic dynamical systems Math. USSRIzvestiya 8 (1974) 177-218.

[7] Burns K., Pugh C. \& Wilkinson A. Stable ergodicity and Anosov flows, preprint.

[8] Burns K. \& Wilkinson A. Stable ergodicity of skew products, preprint. 
[9] Castro A. Backwards inducing and decay of correlations for certain partially hyperbolic maps whose central direction is mostly contracting IMPA Thesis, 1998.

[10] Chernov N. I. Decay of correlations and dispersing billiards, J. Stat. Phys. 94 (1999) 513-556.

[11] Chernov N. I. \& Haskell C. Nonuniformly hyperbolic K-systems are Bernoulli Erg. Th. \& Dyn. Sys. 16 (1996) 19-44.

[12] Dolgopyat D. Limit theorems for partially hyperbolic systems, preprint

[13] Grayson M., Pugh C. \& Shub M. Stably ergodic diffeomorphisms Ann. Math. 140 (1994) 295-329.

[14] Hirsh M., Pugh C. \& Shub M. Invariant manifolds Lect. Notes in Math. 583 Springer-Verlag, Berlin, 1977.

[15] Katok A. \& Hasselblatt B. Introduction to the modern theory of dynamical systems Encyclopedia Math., Appl. 54 (1998) Cambridge University Press.

[16] Katok A.\& Kononenko A.Cocycle stability for partially hyperbolic systems Math. Res. Lett. 3 (1996) 191-210.

[17] Margulis G. A. Certain measures that are connected with U-flows on compact manifolds Func. Anal., Appl. 4 (1970) 62-76.

[18] Margulis G. A. PhD Thesis, Moscow State University, 1970.

[19] Milnor J. On the concept of attractor Comm. Math. Phys. 99 (1985) 177-195 and 102 (1985) 517-519.

[20] Nitica V. \& Torok A. An open dense set of stably ergodic diffeomorphisms in a neighbourhood of a non-ergodic one, to appear in Topology.

[21] Ornstein D. \& Weiss B. On Bernoulli nature of systems with some hyperbolic structure Erg. Th. \& Dyn. Sys. 18 (1998) 441-456.

[22] Palis J. Global view on dynamics to appear in Asterisque.

[23] Palis J., Pugh C. C. \& Robinson R. C. Nondifferentiability of invariant foliations Lecture Notes Math. 468 (1975) 234-240 Springer, Berlin.

[24] Pesin Ya. B. Families of invariant manifolds that correspond to nonzero characteristic exponents Izv. Akad. Nauk SSSR 40 (1976) 1332-1379.

[25] Pesin Ya. B. Characteristic Lyapunov exponents, and smooth ergodic theory Uspehi Mat. Nauk 32 (1977) 55-112.

[26] Pesin Ya. B. \& Sinai Ya. G. Gibbs measures for partially hyperbolic attractors Erg. Th. \& Dyn. Sys. 2 (1982) 417-438.

[27] Pugh C. \& Shub M. Ergodic attractors Trans. AMS 312 (1989) 1-54.

[28] Pugh C. \& Shub M.Stably ergodic dynamical systems and partial hyperbolicity J. Complexity 13 (1997) 125-179.

[29] Pugh C. \& Shub M. Stable ergodicity and Julienne quasi-conformality, preprint.

[30] Ruelle D. A measure associated with Axiom A attractors Amer. J. Math. 98 (1976) 619-654.

[31] Ruelle D. Differentiation of SRB states Comm. Math. Phys. 187 (1997) 227241.

[32] Shub M. \& Wilkinson A. Pathological foliations and removable zero exponents Inv. Math (1999).

[33] Sinai, Ya. G. Classical dynamic systems with countably-multiple Lebesgue spectrum. II Izv. Akad. Nauk SSSR Ser. Mat. 30 (1966) 15-68.

[34] Sinai Ya. G. Gibbs measures in ergodic theory Uspehi Mat. Nauk 27 (1972) 21-64. 
[35] Sinai Ya. G. Stochasticity of dynamical systems in Nonlinear waves, Edited by A. V. Gaponov-Grekhov Nauka, Moscow (1979) 192-211.

[36] Viana M. Multidimensional nonhyperbolic attractors Publ. IHES 85 (1997) 63-96.

[37] Wilkinson A. Stable ergodicity of time-one map of a geodesic flow $\mathrm{PhD}$ Thesis, University of California at Berkeley, 1995.

[38] Young L.-S. Statistical properties of dynamical systems with some hyperbolicity including certain billiards, Ann. Math. 147 (1998) 585-650.

[39] Young L.-S. Recurrence times and rates of mixing, Isr. J. Math 110 (1999) 153-188.

Department of Mathematics, Penn State University, State College, PA 16802

E-mail address: dolgop@math.psu.edu 